\title{
Caminos rurales, consorcios camineros y desarrollo regional en la provincia de Córdoba (Argentina) en la segunda mitad del siglo XX
}

\section{Rural roads, road consortia and regional development in the province of Córdoba (Argentina) in the second half of the 20th century}

\author{
H. Martín Civitaresi \\ Licenciado en Economía, Universidad Nacional de Río Cuarto, \\ Master of Arts en Estudios del Desarrollo, Erasmus University-Institute of Social Studies y \\ Doctor en Historia de la Universidad Nacional de Colombia \\ Profesor Asociado de la Escuela de Economía, Administración y Turismo de la sede \\ andina de la Universidad Nacional de Río Negro, Argentina \\ https://orcid.org/0000-0001-6375-1678 \\ hcivitaresi@unrn.edu.ar \\ Fecha de recepción: 30 de marzo del 2018 \\ Fecha de aceptación: 5 de junio del 2018 \\ Disponible en línea: 27 de junio del 2018
}

Sugerencia de citación: Civitaresi, H. M. (2018). Caminos rurales, consorcios camineros y desarrollo regional en la provincia de Córdoba (Argentina) en la segunda mitad del siglo XX. tiempo\&economía, 5(2), 89-105, doi: http://dx.doi.org/10.21789/24222704.1359

\section{RESUMEN}

Se aborda un problema de acción colectiva a partir del capital social: el mantenimiento de los caminos rurales con consorcios camineros como práctica asociativa a partir de demandas sociales y económicas de infraestructura en un área de la región pampeana Argentina. Se trata del aporte de vecinos y productores a partir de prácticas asociativas para afrontar, junto con los gobiernos provincial y nacional, las obras camineras rurales. Se identifican aspectos facilitadores institucionales, sociales y económicos de trabajo asociativo, como sus aportes al desarrollo rural. 
Palabras clave: historia regional, capital social, acción colectiva, consorcios camineros, historia económica

\section{Códigos JEL: N96}

\section{ABSTRACT}

A problem of collective action based on social capital is addressed: the maintenance of rural roads with road consortia as an associative practice based on social and economic demands of infrastructure in a specific area of the Argentinean Pampas. The article refers to a contribution of neighbors and producers to face, together with the provincial and national governments, rural road works. Institutional, social and economic drivers of associative work are identified as well as their contributions to rural development.

Keywords: Regional history, social capital, collective action, Road Consortia, economic history

JEL Code: N96 


\section{Introducción}

El agro en la economía argentina ha sido importante a lo largo de las distintas etapas en las que se puede dividir su historia. Flichman (1977) sostiene que la cuestión agraria -todos aquellos temas que abarca lo concerniente a la producción del agro- es decisiva por dos razones. La primera es el peso extraordinario que la producción agropecuaria tiene en el sector externo y que condiciona decisivamente el ciclo general de la economía. La segunda razón, es el papel que la producción agropecuaria ha desempeñado en la fijación del valor de la fuerza de trabajo, es decir, del salario real, en el mercado interno ya que proporciona una buena parte de la canasta familiar (bienes salario).

Sin desmerecer la importancia de economías regionales extra pampeanas, como por ejemplo la mendocina con su producción vitivin aícola, la región de la pampa argentina es la que se ha constituido en la base de la estructura económica de la Argentina y, fundamentalmente, en un articulador socioeconómico en torno al cual se estructuraron proyectos nacionales, se dibujaron sujetos sociales en pugna, se instituyeron organizaciones y se implementaron políticas de Estado (Barsky y Gelman, 2001). ${ }^{1}$ En este sentido, el desempeño del agro pampeano debe ser analizado no sólo en términos sectoriales sino también a partir de la organización al interior del propio sector y su relación con el resto de los sectores a lo largo de los procesos históricos nacionales, desde un modelo agroexportador hasta un modelo de Industrialización por Sustitución de Importaciones (Neffa, 1998) o como lo definen Bértola y Ocampo (2010) una "Industrialización dirigida por el Estado".

Este artículo estudia el agro pampeano desde la década de 1950. Si bien se trata de un escenario macrohistórico distinto al del modelo agroexportador, dicha región continuó siendo un actor fundamental como generador de divisas y proveedor de bienes salario a partir de la explotación agropecuaria (Olivera, 2008). Específicamente se hace referencia a la conformación y funcionamiento de la estructura de Consorcios Camineros en la provincia de Córdoba (mapa 1). Es decir, se destaca la capacidad de organización de actores rurales para realizar prácticas asociativas para afrontar, junto con los gobiernos provincial y nacional, diferentes demandas sociales y económicas de infraestructura, particularmente relacionadas a los caminos rurales existentes en una provincia específica.

Se aborda desde una perspectiva de historia regional ya que, de esta manera, se puede tener un marco territorial específico para los resultados de una acción colectiva sobre la base del capital social. Utilizar una perspectiva focalizada en regiones permite transitar de un enfoque macrohistórico a un análisis micro. Es decir, a partir de la historia regional se puede ver que los resultados de las trayectorias históricas no son obtenidos por una cadena de causalidades sistémicas inevitables, sino que hay ciertos márgenes de libertad para que diversos actores interactúen y construyan socialmente un espacio (Gelman, 2007; Girbal-Blacha, 2007, 2013).

1 Para mayor detalle de la historiografía rural pampeana del siglo XX ver Barsky y Djenderedjian (2006), Barsky y Pucciarelli (1997), Barsky, Posada y Barsky (1992), Bonaudo y Godoy (1985), Bonaudo (2006, 2007), Campi (2008), Djenderedjian (2008), Gallo (1983), Girbal-Blacha (2001), Graciano y Lazzaro (2007), Hora (2015), Lattuada (2006), Taylor (1948).

tiempo\&economía

Vol. 5 N. ${ }^{\circ} 2$ - Julio - Diciembre del 2018 


\section{Mapa 1. Mapa político de la provincia de Córdoba}

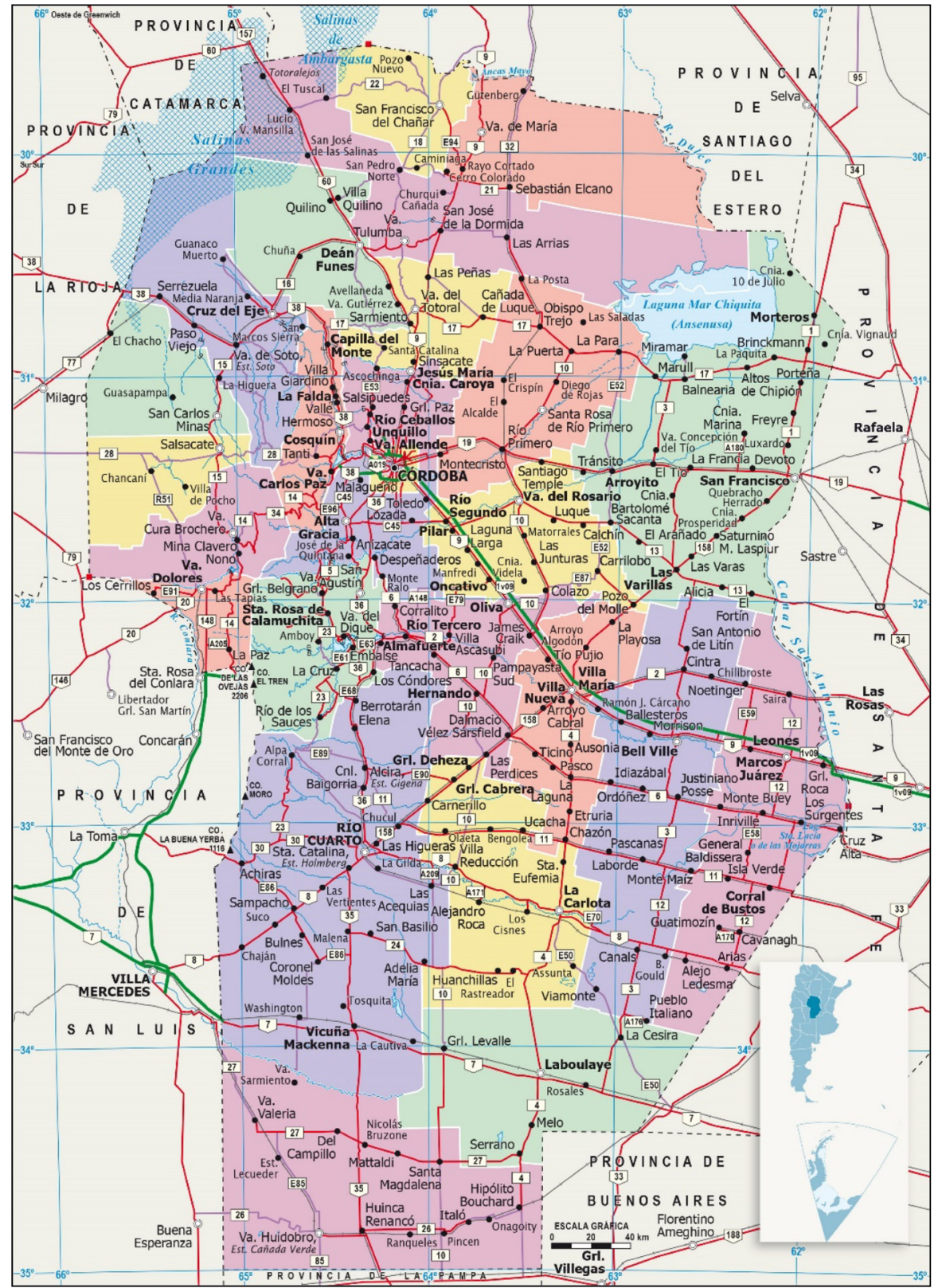

Fuente: Instituto Geográfico Nacional. http://www.ign.gob.ar/AreaServicios/Descargas/Mapas

El artículo se organiza de la siguiente manera: en la próxima sección se destacan las características y la importancia de los caminos rurales para afianzar el desarrollo socioeconómico regional. Posteriormente, en las dos secciones siguientes se representan los consorcios camineros, primero como una estrategia fomentada por distintas escalas de gobierno y luego como una estrategia de acción colectiva sobre la base del capital social. Luego, se presentan reflexiones finales sobre las sinergias entre el Estado, para la promoción y el financiamiento de los consorcios camineros, y la capacidad de organización y colaboración de productores agropecuarios para el mantenimiento de la red vial rural en la provincia de Córdoba. 


\section{Caminos rurales y el desarrollo socioeconómico regional}

Los caminos rurales, también denominados caminos vecinales, conforman un sistema vinculante con vías principales y secundarias (rutas nacionales o provinciales) que sirven a actividades agropecuarias, mineras y forestales, etc., que se desarrollan en zonas aledañas (Álvarez, 1979). Se trata de caminos de vinculación sin pavimentación que tienen por objetivo la conexión de pequeñas localidades para garantizar los derechos económicos, sociales y culturales de las personas como así también la articulación de explotaciones agropecuarias con las rutas provinciales y nacionales para la extracción de su producción y el acceso a insumos que se requieren para desarrollar la actividad productiva. Es decir, los caminos rurales resultan fundamentales para el desarrollo socioeconómico rural no sólo por ser las primeras vías alimentadoras hacia el destino final exportador de la producción agropecuaria, sino porque también tienen una importante función como articuladores clave en términos económicos, sociales y culturales (Fouracre y Dyson, 2006; Lordi y Regazzoli, 2011).

Las redes de caminos rurales siempre han sido más extensas que las redes de caminos primarios y provinciales, con frecuencia duplican o triplican su tamaño, pero soportan menos tránsito (apenas el 10\% del tránsito en vehículo/kilómetro) por lo que su mantenimiento ha tenido menos prioridad que el de redes de caminos principales. Esto no quiere decir que su falta de mantenimiento no haya representado un problema para el desarrollo socioeconómico rural; su descuido provoca costos adicionales de transporte (p. ej. imposibilidad de sacar la producción oportunamente, recorridos más extensos, etc.) como también problemas en términos de bienestar social.

Esta problemática ha sido tenida en cuenta tanto por los gobiernos nacional y provinciales como por los productores agropecuarios y vecinos de localidades rurales como principales involucrados. En el primer caso, la búsqueda de una solución se dio desde el poder legislativo nacional, por iniciativa del Ing. Emilio Mitre, a través de la sanción de la Ley Mitre. ${ }^{2}$ En el artículo 8 se disponía una contribución del 3\% de los ingresos de las concesiones ferroviarias para la construcción de caminos de acceso a estaciones ferroviarias permitiendo aliviar a las provincias en esa tarea ya que la actividad económica que producían los ferrocarriles era creciente. Dichos fondos fueron administrados por la Comisión del Fondo de Caminos (Álvarez, 1957; Scalabrini Ortiz, 1974; López, 2007).

Desde la implementación de la Ley Mitre, Ley n 5.315/1907, siempre se buscó la solución a los problemas de conservación de los caminos rurales a partir de la participación activa de los propios interesados en el uso del camino (Maldonado Torres, 1989). Concretamente se buscaba involucrar a vecinos de las localidades rurales y productores agropecuarios, pero también a los gobiernos locales tales como municipalidades, en el caso de las ciudades, o de comisiones de fomento para poblaciones menores. Existen ejemplos a lo largo de todo el territorio argentino que demuestran esta situación. Uno de ellos es el caso de los consorcios camineros del Touring Club Argentino, conformados a fines de 1931 para solucionar los problemas de los

2 López (2007) y Regalsky (2007) estudian los antecedentes de la Ley Mitre de 1907 desde dos enfoques complementarios. El primero destaca aspectos políticos y diferencias de perspectivas de los grupos sociales vinculados al gobierno. El segundo destaca los intereses de las empresas ferroviarias y la necesidad de encontrar algunos puntos de acuerdo.

tiempo\&economía

Vol. 5 N. ${ }^{\circ} 2$ - Julio - Diciembre del 2018 
caminos rurales el Territorio Nacional de La Pampa (Ayala y Gette, 2007). ${ }^{3}$ También se observa en las provincias de Santa Fe y Entre Ríos entre 1931-1932; allí, los consorcios vecinales trabajaron asistidos y financiados por el gobierno provincial (Álvarez, 1957; 1979).

Los casos de Santa Fe y Entre Ríos, junto con la provincia de Buenos Aires, son citados en los considerandos del Boletín Oficial de la República Argentina de 1931 -1ª sección, en donde se publica la creación del Directorio Central de Consorcios Camineros. Allí se dice: "algunos gobiernos provinciales [Buenos Aires, Santa Fe y Entre Ríos] y comunales han encarado estos asuntos con resultados excelentes que podrían ser de mayor importancia con el aporte del Gobierno de la Nación".

El Directorio Central de Consorcios Camineros tenía por objetivo "la construcción, mejoramiento o conservación de caminos de tierra, vigilancia de los caminos firmes, cumplimiento de las reglamentaciones de tráfico y colocación y conservación de señales camineras". Sin embargo, más allá de este marco legal y el trabajo conjunto con comisiones de fomento y productores en algunas provincias pampeanas y territorios nacionales, se puede leer en Halperin (2005, p. 315) un texto de Alejandro Bunge de 1930 en la Revista de Economía Argentina que este tipo de medidas no terminaron de transformar el mal estado de los caminos rurales:

Existe una evidente desproporción entre la magnífica y extensa red ferroviaria con que cuenta la Argentina y los caminos de afluencia a sus estaciones. En tanto contamos con 38 mil km de ferrocarriles, sólo poseemos unos 25 mil km de caminos, sumado los firmes y los de tierra, bien acondicionados, lo cual resulta en gran desproporción con la extensión ferroviaria. Este hecho limita la zona de afluencia de nuestros ferrocarriles y encarece el acarreo de nuestros productos agrícolas y ganaderos hasta las estaciones. Es de lamentar que ni siquiera los fondos de nuestra Ley Mitre hayan sido debidamente aplicados a su específica finalidad, es decir, a la construcción y conservación de caminos de afluencia a las estaciones.

Se ha difundido la creencia de que el camino y el ferrocarril son competidores desde la difusión del automotor. Esto, que excepcionalmente resulta exacto tratándose de pequeños ramales ferroviarios destinados a proveer tráfico a las líneas troncales, no lo es tratándose del conjunto general de los dos sistemas de transportes. Ellos se complementan. Y es, precisamente, porque nosotros carecemos aún de los pequeños ramales ferroviarios de afluencia a las grandes líneas transportadoras, que la función más importante que ha de desempeñar el camino en la Argentina será la de substituir, con el automotor, el pésimo y costosísimo acarreo actual. Los beneficios del riel 'demasiado lejos' del ferrocarril para poder transportar con beneficio la producción a los grandes centros consumidores y a los grandes puertos.

3 Una vez desplazada la población originaria con la campaña de 1879, los espacios de frontera fueron organizados en Territorios Nacionales, entidades político administrativas dependientes del gobierno nacional, carentes de autonomía y con habitantes que portaban derechos políticos restringidos. A través de la Ley n 954/1878 se dispuso la creación de la Gobernación de la Patagonia con Viedma como Capital y posteriormente, con la Ley n 1532/1884, se ordenó en unidades administrativas más pequeñas creando los Territorios Nacionales de Neuquén, Río Negro, Chubut, Santa Cruz y Tierra del fuego. Se postergó la creación de provincias con el argumento que se preservaban esos espacios para que no sufrieran los procesos disolutivos que habían sufrido las provincias antiguas en la primera mitad del siglo XIX. Era necesario un acompañamiento por parte del gobierno nacional hasta que esos territorios pudieran incorporarse plenamente como provincias autónomas (Ruffini, 2003). 
Es por esta razón que consideramos de importación secundaria los grandes caminos espectaculares destinados a cruzar extensas regiones de la República y que insumirán, cualquiera de ellos decenas de millones. Creemos necesario se tenga en todo momento presenta la conveniencia económica de 'coordinar' ambos medios de comunicación como la única forma de abaratar los transportes hasta hoy caros y a veces prohibitivos en ciertas zonas del país, que aun cuando están lejos del riel, son aptas para la producción exportable.

A partir de la década de 1930 los fondos de la Ley Mitre, y sus obras camineras estuvieron a cargo, primero, de la Dirección de Puentes y Caminos y, posteriormente, con la sanción de la Ley $n^{\circ} 11.658$ en 1932, de la Dirección Nacional de Vialidad (DNV) la cual también disponía de los fondos obtenidos por un impuesto de 2 centavos por cada litro de nafta expedido (Ayala y Gette, 2007). A partir de la creación de la DNV, y siguiendo lo planteado en la Ley Nacional, se fueron creando las distintas DPVs como entes autárquicos administrativa y financieramente; con potestad para proyectar, construir y conservar la red vial. En Córdoba, la DPV fue creada con la sanción de la Ley n³ 3.648 de 1935 (ACCPC, 2010).

El marco legal en torno a las áreas administrativas de los gobiernos nacional y provinciales a cargo de la red de caminos estaba dispuesto a fines de la década de 1930. No obstante, la situación para la red de caminos rurales continuaba siendo desfavorable. Por un lado, el crecimiento del transporte automotor y la pérdida de carga del ferrocarril plantearon la necesidad de un mayor desarrollo de caminos alimentadores de la red de caminos nacionales. Por otro, no se implementó una política estable para la construcción y mantenimiento de este tipo de caminos durante la década de 1940 (AAC, 2003). Para la década de 1950, se reconocía la importancia del sistema vial terciario para el desarrollo socio-económico del país. En revistas especializadas como la Revista Carreteras de la Asociación Argentina de Carreteras se puede leer en sus editoriales este reconocimiento:

Este tipo de caminos no son ramales del sistema vial, sino, por el contrario, sus raíces. Ellos 'alimentan' al aparato y justifican su existencia. De la lozanía y vigor de los caminos vecinales depende, en último análisis, la eficiencia del mecanismo intercomunicador. Extendiendo, aún más, el concepto, puede afirmarse que un sistema de carreteras troncales tiene como misión fundamental enlazar las distintas redes de caminos vecinales. De allí se sigue que éstos son la parte esencial que incide, básicamente, en el desarrollo de la economía nacional (Hummet, 1956)

Pero el problema del escaso desarrollo de la red de caminos rurales aún no se afrontaba de manera eficaz. Se requería de una mayor participación de los productores agropecuarios y vecinos de localidades rurales como los principales actores involucrados en su mantenimiento. También se necesitaba reflotar la creación de organizaciones representativas, los consorcios camineros, para aportar no sólo una contribución pecuniaria sino también su conocimiento y, sobre todo, la fiscalización de las obras realizadas.

\section{Consorcios camineros fomentados por los gobiernos}

Desde mediados de la década de 1950, los consorcios camineros han colaborado con los gobiernos, a través de las distintas Direcciones Provinciales de Vialidad (DPVs), para la

tiempo\&economía

Vol. 5 N. 2 - Julio - Diciembre del 2018 
Caminos rurales, consorcios camineros y desarrollo regional en la provincia de Córdoba (Argentina) en la segunda mitad del siglo XX

doi: https://doi.org/10.21789/24222704.1359

ejecución de trabajos viales, construcción, conservación y mejoramiento de los caminos de la red terciaria. Además, tuvieron la posibilidad de extender sus actividades hacia la red secundaria (carreteras que conectan ciudades de importancia media) o también realizar contrataciones con organizaciones privadas, siempre que no desvirtuara su objetivo principal.

En el caso de la provincia de Córdoba, para mediados del siglo XX, el problema de la intransibilidad de los caminos era similar al resto de la región pampeana. Debido a ello, y utilizando el marco legal vigente de la época, un par de diputados provinciales presentaron un proyecto de ley en 1952. Allí se establecía, en el primer artículo, la creación de “...consorcios camineros en todas las ciudades y pueblos de la provincia con más de doscientos habitantes que serán instituciones de Derecho Público y tendrán capacidad para actuar privada y públicamente, de conformidad a la presente Ley" (ACCPC, 2010). Fue aprobado como Ley, pero por deficiencias presupuestarias para su aplicación nunca pudo implementarse.

Sin embargo, el caso de los caminos rurales cordobeses, junto con situaciones similares en el resto de las provincias pampeanas, resultaron un claro manifiesto de una necesidad concreta y derivó en la sanción del Decreto Ley Nacional № 9875 en 1956 que estableció un "Plan de Caminos de Fomento Agrícola" para todo el territorio de la República Argentina. Este Plan permitió la posterior formación, control, apoyo y asesoramiento de consorcios camineros, canalizando formalmente la cooperación de vecinos productores agropecuarios en la conservación de ese tipo de caminos. Este marco institucional nacional y provincial, y el apoyo económico asociado, resultaron fundamentales. Diez años después de la puesta en marcha del Plan de Caminos de Fomento Agrícola se habían creado en el país cerca de tres mil consorcios camineros en el país que recibieron asesoramiento técnico, equipos y hasta el $70 \%$ de financiación de las obras realizadas.

Si bien todas las provincias habían sido incluidas en los beneficios del Plan, los consorcios camineros se habían distribuido desigualmente a lo largo del territorio nacional siguiendo patrones de acumulación propias de los existentes en el sector agropecuario. Gran parte estaba en las tres mayores provincias de la región pampeana (Buenos Aires, Santa Fe y Córdoba). Asimismo, si se calculan indicadores tales como el número de obras por consorcio o la longitud de kilómetros mantenidos por consorcio es posible identificar marcadas diferencias entre estas tres provincias pampeanas (tabla 1).

La provincia de Córdoba muestra para 1967 un dinamismo particular. La cantidad de obras por consorcio caminero es superior en esta provincia (3.06) si se compara con otras dos provincias importantes de la región pampeana como Santa Fe (1.76) y Buenos Aires (1.22). Algo similar ocurre con la cantidad de kilómetros por consorcio caminero. En diez años, cada uno de los consorcios camineros en promedio había construido 205 kilómetros en el caso de Córdoba, 48 y 52 kilómetros para el caso de Santa Fe y Buenos Aires, respectivamente.

Dos actores, que se detallan a continuación, parecen ser clave en el buen desempeño de los consorcios camineros cordobeses. El primero es la DPV la cual, además de fiscalizar contable, patrimonial y administrativamente a los consorcios, también proveía de asesoramiento y apoyo financiero. El segundo, cronológicamente posterior, es la Asociación de Consorcios Camineros de la Provincia de Córdoba (ACCPC) por su rol de intermediación entre los consorcios y el gobierno provincial. 
Tabla 1. Consorcios camineros. Síntesis de la labor realizada hasta julio de 1967

\begin{tabular}{|c|c|c|c|c|c|c|c|c|}
\hline Distritos & $\begin{array}{l}\text { Consorc. } \\
\text { camineros }\end{array}$ & $\begin{array}{c}\text { Obras } \\
\text { aprobadas }\end{array}$ & $\begin{array}{l}\text { Obras aprob/ } \\
\text { const.camin }\end{array}$ & $\begin{array}{l}\text { Longitud } \\
\mathrm{Kms}\end{array}$ & $\begin{array}{l}\mathrm{Kms} / \text { cons. } \\
\text { camin }\end{array}$ & $\begin{array}{l}\text { Presupuesto } \\
m \$ n \text { en miles }\end{array}$ & $\begin{array}{l}\text { Aportes } \\
\text { DNV (\%) }\end{array}$ & $\begin{array}{c}\text { Aporte Cons. } \\
\text { Camin.(\%) }\end{array}$ \\
\hline Bs Aires & 845 & 1,033 & 1.22 & 43,938 & 52 & $1,006,605$ & $71 \%$ & $29 \%$ \\
\hline Santa fe & 358 & 630 & 1.76 & 17,340 & 48 & 249,842 & $72 \%$ & $28 \%$ \\
\hline Córdoba & 234 & 716 & 3.06 & 47,957 & 205 & 376,705 & $72 \%$ & $28 \%$ \\
\hline San Juan & 173 & 131 & 0.76 & 3,017 & 17 & 142,039 & $68 \%$ & $32 \%$ \\
\hline Chaco & 170 & 167 & 0.98 & 2,663 & 16 & 86,837 & $72 \%$ & $28 \%$ \\
\hline La Pampa & 126 & 256 & 2.03 & 21,463 & 170 & 132,807 & $67 \%$ & $33 \%$ \\
\hline Mendoza & 115 & 113 & 0.98 & 6,601 & 57 & 230,536 & $61 \%$ & $39 \%$ \\
\hline Entre Ríos & 108 & 81 & 0.75 & 1,269 & 12 & 113,712 & $74 \%$ & $26 \%$ \\
\hline S. d Estero & 104 & 154 & 1.48 & 3,688 & 35 & 193,586 & $71 \%$ & $29 \%$ \\
\hline Corrientes & 101 & 114 & 1.13 & 1,979 & 20 & 129,943 & $68 \%$ & $32 \%$ \\
\hline Tucumán & 97 & 95 & 0.98 & 4,979 & 51 & 240,562 & $69 \%$ & $31 \%$ \\
\hline San Luis & 93 & 83 & 0.89 & 7,892 & 85 & 93,603 & $70 \%$ & $30 \%$ \\
\hline Salta & 79 & 78 & 0.99 & 1,191 & 15 & 168,331 & $67 \%$ & $33 \%$ \\
\hline Catamarca & 47 & 37 & 0.79 & 333 & 7 & 53,742 & $61 \%$ & $39 \%$ \\
\hline Jujuy & 45 & 62 & 1.38 & 259 & 6 & 64,590 & $63 \%$ & $37 \%$ \\
\hline Río Negro & 45 & 260 & 5.78 & 8,740 & 194 & 110,140 & $72 \%$ & $28 \%$ \\
\hline Chubut & 36 & 35 & 0.97 & 1,502 & 42 & 63,277 & $75 \%$ & $25 \%$ \\
\hline Misiones & 36 & 76 & 2.11 & 9,650 & 268 & 86,576 & $73 \%$ & $27 \%$ \\
\hline La Rioja & 24 & 14 & 0.58 & 175 & 7 & 33,303 & $83 \%$ & $17 \%$ \\
\hline T. d Fuego & 23 & 15 & 0.65 & 184 & 8 & 16,557 & $70 \%$ & $30 \%$ \\
\hline Formosa & 21 & 46 & 2.19 & 933 & 44 & 59,725 & $76 \%$ & $24 \%$ \\
\hline Neuquén & 19 & 35 & 1.84 & 844 & 44 & 48,416 & $74 \%$ & $26 \%$ \\
\hline Santa cruz & 17 & 13 & 0.76 & 1,186 & 70 & 33,932 & $72 \%$ & $28 \%$ \\
\hline Totales & 2,916 & 4,244 & 1.46 & 189,785 & 65 & $3,735,366$ & $70 \%$ & $30 \%$ \\
\hline
\end{tabular}

\section{El plan de caminos de fomento agrícola de la provincia de Córdoba}

La Provincia de Córdoba adhirió al Plan de Caminos de Fomento Agrícola a través del Decreto $\mathrm{N}^{\circ} 645$ de 1956 determinando que la DPV fuera el órgano que ejerciera el control y fiscalización contable, patrimonial y administrativa y quien brindara asesoramiento (Maldonado, 1989; ACCPC, 2012, 2015). Dos años después, en la descripción sobre la situación de vialidad de la provincia de Córdoba en la revista Carreteras, se destacaba que la promoción en la formación de consorcios camineros fue eficaz, fortaleciendo 117 grupos de pequeños y medianos contratistas y ampliando la órbita de sus tareas propias (Molina, 1958). Sin embargo, el impacto de su accionar todavía resultaba incipiente. De hecho, en las zonas agropecuarias de la provincia subsistían algunos problemas. Por ejemplo, en 1958 se mencionaba que muchos 
caminos rurales se encontraban por debajo del nivel de los terrenos laterales. Esto implicaba que las trazas se transformaran en canales con agua en épocas de lluvia (García, 1958).

La construcción institucional en torno al manejo de caminos rurales continuó en 1973 con la creación de la ACCPC. La misma fue reconocida por DPV como el organismo representativo de los consorcios camineros de todo el territorio provincial, además de cumplir otras funciones como la comercialización de insumos, productos y herramientas para los consorcios, a mejor precio que en el mercado. Para 1989, 275 consorcios camineros cubrían 37.200 km de la red terciaria (de un total de $74.600 \mathrm{~km}$ ) y atendían también a $11.395 \mathrm{~km}$ de caminos de la red secundaria (de $15.795 \mathrm{~km}$ ). Es decir que, del total de caminos con servicio de conservación permanente (52.995 km), los consorcios camineros atendían a 48.595 km (91,7\%) (Picca, 2016).

Los ingresos de los consorcios camineros provenían de las cuotas sociales normales y extraordinarias que realizaban los socios y adherentes. En zonas donde existían cooperativas que centralizaban la comercialización de productos, dichas cooperativas realizaban las retenciones y transferencias al consorcio. En zonas con reducida capacidad económica, algunos consorcios reemplazaban el aporte de la cuota por trabajo personal de los socios.

Otra fuente de ingresos provenía de obras realizadas por el consorcio. La DPV asignaba a cada consorcio anualmente, en un plan de trabajo, una cantidad de kilómetros a conservar con un precio unitario por kilómetro conforme a las características topográficas de la zona. De esta manera, cada consorcio recibía aportes mensuales desde el gobierno provincial. ${ }^{4}$ Asimismo, recibía ingresos de subsidios o donaciones en efectivo, equipos y materiales de instituciones públicas o privadas y de fondos que le asignaba la DPV de sus propios recursos. En este último caso, el marco legal establecía una coparticipación federal del 35\% de lo recaudado, con afectación específica a caminos vecinales de al menos el $50 \%$ de lo que le correspondía a cada provincia (Maldonado, 1989).

Este financiamiento duró hasta 1977 cuando el gobierno de facto eliminó los recursos que se coparticipaban para el desarrollo del Plan de Fomento Agrícola de 1956 y sólo existieron esfuerzos provinciales y de los propios consorcios camineros (AAC, 2003). A partir de allí, la cantidad de caminos rurales conservados por consorcios camineros se incrementó a un ritmo menor al existente antes de la última dictadura. Lo mismo sucedió con la cantidad de consorcios, que se mantuvo relativamente estable. En la actualidad, $56.700 \mathrm{~km}$ de caminos de tierra existentes en la provincia son conservados por 289 consorcios camineros organizados en 19 regionales (mapa 2), cada una de las cuales está conformada por un promedio de 15 consorcios camineros (Picca, 2016).

4 Por ejemplo, el principal ingreso del Consorcio Caminero $n^{\circ} 72$ de la localidad de General Cabrera, fue la certificación y pago a cargo de la DPV por el mantenimiento de la red secundaria, terciaria y obras de afirmado y enarenado. Las certificaciones no eran periódicas sino eventuales. Una vez revisadas las obras se realizaba el pago, que podía ser trimestral o cuatrimestral. Por otro lado, el consorcio colaboraba en obras específicas del gobierno provincial. Por ejemplo, entre los años 1988 y 1991, la extensiva siembra de maní en la zona provocó grandes crecientes que llevaron a cubrir varias zonas de la localidad además de dejar los caminos intransitables e irrecuperables. En este caso, desde el Consorcio se gestionaron obras, con el correspondiente apoyo financiero de la provincia, como el canal del camino Tegua (hoy ruta provincial E90) y el canal sobre los caminos transformados, ya que se tuvo que realizar un nuevo trazado, con sus respectivos puentes, ambos a los ingresos de General Cabrera, sobre la ruta nacional 158. 
Mapa 2. Regionales de Conservación de caminos de tierra

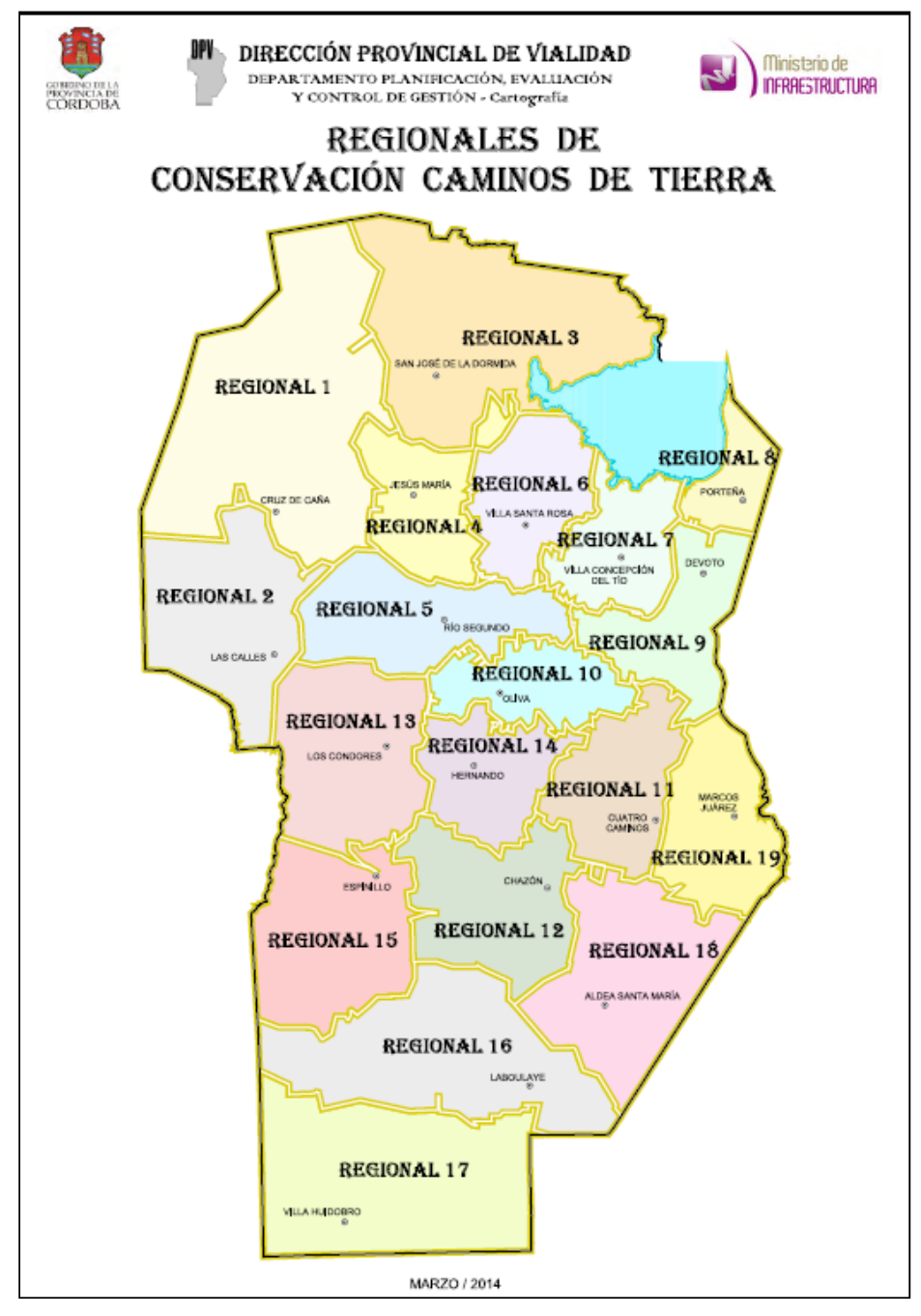

Fuente: DPV (2014)

\section{Consorcios camineros y la acción colectiva sobre el capital social}

Más allá de las normas administrativas dictadas por la Dirección Nacional de Vialidad, el papel de la DPV y el rol de intermediación entre los consorcios y el gobierno provincial de la ACCPC, es necesario destacar la capacidad de organización y colaboración de los productores rurales para poder conformar los consorcios y realizar las obras. Es decir, a un factor institucional formal se le concatena otro asociado al trabajo colectivo. Maldonado sintetiza este último factor de la siguiente manera:

En cuanto a la filosofía de vida que debe imperar en los hombres del pueblo y del campo para que subsista el sistema, podemos decir lo siguiente: a) el trabajo colectivo hace nacer espontáneamente el 'espíritu de solidaridad', lo que constituye sin duda un patrimonio social de invaluable transcendencia para la comunidad; b) los hombres que integran las comisiones directivas de los consorcios camineros son aquellos que han sabido desarrollar virtudes tales como profunda vocación de servicio a la comunidad, capacidad de trabajar por el bien 
sin perseguir fines de lucro, capacidad de aceptar la pluralidad política y/o religiosa entre los consorcistas, conciencia de que hay que servir y aportar para recibir un servicio, predisposición para sacrificar el bienestar personal en beneficio de la comunidad y fortaleza y serenidad para soportar ingratitudes (Maldonado, 1989, p. 14).

En síntesis, fueron dos los aspectos facilitadores que permitieron el trabajo asociativo. Uno de carácter institucional, a partir del rol de los gobiernos de distintas escalas para fomentar la creación de consorcios camineros (detallado en las secciones anteriores); el otro socio-económico, a partir de los acuerdos formales o informales y redes relacionales de productores rurales.

En términos conceptuales, ambos aspectos facilitadores se podrían vincular al concepto de capital social. En un sentido amplio, el capital social hace referencia a todas aquellas situaciones en las que un grupo de actores muestra una relativa capacidad a cooperar para alcanzar determinados objetivos comunes sobre la base de un conjunto de normas y valores compartidos (Atria, Siles, Arriagada, Robison y Whiteford, 2003).

Por su intensivo uso multidisciplinario no existe una definición ampliamente aceptada, sin embargo, a partir de los aportes de distintos autores (Woolcock, 2000; Atria et al., 2003; Bagnasco, Piselli, Pizzorno, y Trigilia, 2003; Ostrom y Ahn, 2003; Valdemarca, 2006) se puede elaborar una definición ad hoc operativa que involucre sus componentes básicos. Por lo tanto, se denomina capital social a la capacidad de organización de una sociedad a partir de instituciones o normas y redes relacionales que facilitan la acción colectiva en procesos de desarrollo socioeconómico local.

En la definición surgen los dos aspectos facilitadores que se mencionaron en la conformación de los consorcios camineros: las instituciones (formales o informales) y las redes relacionales. Desde un punto de vista conceptual, se trata de dos determinantes que dan sustento al concepto de capital social.

Para las primeras, las instituciones, North (1993) pone énfasis en su interacción con las organizaciones para explicar procesos de cambio institucional. Las instituciones pueden ser creadas, como las normas formales (p.ej. leyes), o bien pueden evolucionar a lo largo del tiempo como las costumbres o códigos de conducta informales, generalmente no escritos, que subyacen y complementan a las reglas formales. Por otro lado, las organizaciones son grupos de individuos con identidad común (asociaciones civiles, grupos sociales, partidos políticos, empresas, etc.) que proporcionan estructura a su interacción a partir de los objetivos que persiguen, de la combinación de sus aptitudes y estrategias y de la coordinación dentro del marco institucional donde operan. A medida que las organizaciones van cambiando, se va alterando también la evolución del marco institucional y esto influye en el desarrollo socioeconómico de una sociedad. El énfasis de North en la interacción entre instituciones (normas), organizaciones y cambio institucional sirve para explicar cómo la dimensión institucional resultó clave para el surgimiento de los consorcios camineros.

Las segundas, las redes relacionales entre actores, son las que dan forma a una sociedad en un territorio (Atria et al., 2003). La mayoría de los espacios socio-productivos de un territorio nacen como proyectos colectivos en respuesta a necesidades comunes, desarrollando y/o alcanzando diferentes niveles de asociativismo, diversos mecanismos de vinculación entre ellos, y logrando impacto y visibilización en sus comunidades de origen. A partir de estas experien- 
cias e intervenciones se van creando redes o tramas de interacción ancladas al territorio. Lattuada (2006) afirma que los procesos asociativos ayudan a estimular iniciativas, a movilizar y potenciar recursos y capacidades existentes en el territorio. Rofman y Villar (2007) destacan la importancia que tienen las redes de relaciones que vinculan a sujetos y organizaciones, focalizando en la potencialidad que tiene la propia trama o la relación para incidir en la ampliación de las capacidades o recursos de los sujetos que las conforman. En este sentido, un determinado contexto territorial resulta rico en capital social cuando sus actores individuales o colectivos (asociaciones, organizaciones, cooperativas, etc.) están implicados en redes relacionales; una vez establecidas, las redes son circuitos por donde se intercambia información y confianza (Bagnasco et al., 2003).

Las instituciones y las redes relacionales crean incentivos para generar confianza al establecer mecanismos de recompensas y castigos o, indirectamente, proporcionando información, asesoría técnica, entre otros. Dichas instituciones y redes permitieron ir conformando los distintos consorcios camineros a partir de la colaboración voluntaria de los vecinos de cada pueblo o colonia con distintas formas de participación: consorcistas socios, con voz, voto y con capacidad de ser elegido miembro de la Comisión Directiva; consorcistas adherentes, con voz pero no puede ser electos para funciones directivas y una 'persona de representación necesaria' provenientes de la Municipalidad que cumplía el rol de nexo entre el gobierno local y el consorcio caminero. Se trataba de una estrategia colaborativa con una organización administrativa similar a la estructura de gobierno de las cooperativas: una Asamblea General, una Comisión Directiva, un Comité de presidencia (conformado por dos personas, presidente de la Comisión Directiva y algún secretario, y que puedan tomar decisiones de urgencia y conveniente) y una Comisión Revisora de Cuentas (Maldonado, 1989; Picca, 2016).

\section{Reflexiones finales}

La trayectoria histórica de la provincia de Córdoba no fue sólo consecuencia de una cadena inevitable de causalidades macrohistóricas y de condiciones agroecológicas. Los actores rurales tuvieron cierto margen de libertad para interactuar e influir en la construcción social de un espacio dinámico en términos de desarrollo socioeconómico.

Es indudable que la presencia de buenos caminos rurales representaba un mejoramiento de la calidad de vida de la población rural, traducida en beneficios económicos y sociales. Además del evidente efecto sobre la disminución en los costos de transporte de la producción de productos agropecuarios y la valorización de predios, la conectividad asociada a una red de caminos vecinales permitía un acceso más fluido a servicios esenciales como salud, educación, etc.; consolidación del asentamiento de la población en zonas rurales y, como consecuencia de eso, reducción de la migración interna. Asimismo, posibilitaba una mayor interrelación política, social e institucional de localidades rurales (AAC, 2003). El transporte y las organizaciones que lo facilitaban tienen un impacto directo sobre el proceso de construcción social del territorio, donde cobran relieve la historia regional, las estrategias de los actores y sus proyectos, las iniciativas integradas, las regulaciones y la coordinación (Blanco, 2010).

Hay dos factores concatenados que explican el origen y funcionamiento de los consorcios camineros. El primero tiene que ver con que la Dirección Nacional de Vialidad dictó normas

tiempo\&economía

Vol. 5 N. ${ }^{\circ} 2$ - Julio - Diciembre del 2018 
administrativas que le imprimieron agilidad a la administración pública y la DPV apoyaba técnica y financieramente a los consorcios camineros. El segundo está vinculado al hecho de que las obras fueron realizadas directamente por los consorcios, es decir, por los propios interesados. De esta manera, se puede interpretar que un consorcio caminero se trata de una acción colectiva asociada al mantenimiento de los caminos rurales que se desarrolló sobre la base de un capital social que se construyó con dos factores determinantes. Por un lado, un conjunto de normas planteadas desde los gobiernos nacional y provincial, junto con la promoción y el financiamiento. Por otro, con el capital social de los productores agropecuarios, no sólo en la conformación de los consorcios camineros sino también en la construcción de redes interorganizacionales (ACCPC, ACC) que permitieron generar confianza y operatividad en las interacciones.

De manera que se puede generalizar que las sinergias entre el Estado, en cualquiera de sus escalas, y aquellos grupos sociales organizados pueden dividirse en dos: complementariedad e imbricación. En la primera, el Estado crea las condiciones para que las organizaciones sociales se desarrollen, garantizando derechos sociales y políticos a partir del reconocimiento legal de organizaciones como los consorcios camineros. En la segunda, existe una interrelación directa entre la organización pública y las organizaciones sociales. Los consorcios camineros se fueron construyendo a partir de un conjunto de normas planteadas desde los gobiernos nacional y provincial, junto con la promoción y el financiamiento, pero también con la capacidad de organización propia de los productores agropecuarios.

Finalmente, y a modo de cierre, debe considerarse que el aporte principal de este artículo es el tratamiento de otras dimensiones de análisis en el marco de la historia rural pampeana sin pretender brindar una generalización de una problemática sino más bien abrir una ventana analítica diferente. En otras palabras, sin desconsiderar la importancia de las condiciones edafoclimáticas, las políticas públicas a escala nacional o las vicisitudes de los mercados internacionales de commodities agropecuarias como factores clave para el desarrollo rural de la región pampeana, se resalta aquí la capacidad de organización de actores locales para interactuar e influir en la construcción social de un espacio dinámico en términos de desarrollo socioeconómico.

\section{Referencias}

Álvarez, M. (1957). Organización permanente de los caminos vecinales. Revista Carreteras, 12, octubre-diciembre.

Álvarez, M. (1979). Los caminos de la red terciaria. Revista Carreteras, 91, julio-septiembre.

Asociación Argentina de Carreteras - AAC. (1967). La acción oficial + iniciativa privada = Plan de caminos de fomento agrícola. Revista Carreteras, 91, julio-septiembre.

Asociación Argentina de Carreteras - AAC. (2003). Plan de Caminos Rurales. Revista Carreteras, 169, marzo.

Asociación de Consorcios Camineros de La Provincia de Córdoba - ACCPC (5 de octubre de 2010). Día del Camino. Disponible en: //www.accpc.com.ar/index.php?option=com_content\&view=article\&id=27\%3A5-de-octubre-dia-del-camino\&ltemid=83 
Asociación de Consorcios Camineros de La Provincia de Córdoba - ACCPC (Junio/julio de 2012). Los caminos rurales. Disponible en: http://www.accpc.com.ar/images/accpc_pict/ PDF/revistas/accpc_072012.pdf

Asociación de Consorcios Camineros de La Provincia de Córdoba - ACCPC (2015). Consorcios camineros en marcha: General Cabrera. Revista Caminos Rurales, 28, abril-mayo.

Atria, R., Siles, M., Arriagada, I., Robison, L. y Whiteford, S. (2003). Capital social y reducción de la pobreza en América Latina y el Caribe: en busca de un nuevo paradigma. Santiago de Chile: CEPAL.

Ayala, J. y Gette, S. (2007). Historia del camino y los transportes en La Pampa Territoriana (18441951). En Di Liscia et al. (eds.), Al oeste del paraíso. La transformación del espacio natural, económico y social en la Pampa Central, siglos XIX-XX (pp. 107-119). La Pampa: Instituto de Estudios Socio-Históricos, Universidad Nacional de la Pampa, Miño y Dávila Editores.

Bagnasco, A., Piselli, F., Pizzorno, A. y Trigilia, C. (2003). El capital social. Instrucciones de uso. Buenos Aires: Fondo de Cultura Económica.

Barsky, O. y Djenderedjian, J. (2006). Problemas y desafíos de una gran cuestión abierta. La historiografía agraria pampeana del siglo XX. En J. Gelman (comp.), La historia económica argentina en la encrucijada. Balances y perspectivas (pp. 247-267). Buenos Aires: Prometeo Libros - Asociación Argentina de Historia Económica.

Barsky, O. y Pucciarelli, A. (1997). El agro pampeano. El fin de un período. Buenos Aires: Universidad de Buenos Aires.

Barsky, O., Posada, M. y Barsky, A. (1992). El pensamiento agrario argentino. Buenos Aires: Centro Editor de América Latina.

Barsky, O. y Gelman, J. (2001). Historia del Agro Argentino: desde la conquista hasta comienzos del siglo XXI. $1^{\text {a }}$ ed. Buenos Aires: Sudamericana.

Bértola, L. y Ocampo, J. A. (2010). Desarrollo, vaivenes y desigualdad una historia económica de América Latina desde la independencia. Secretaría General Iberoamericana. Disponible en: http://www20.iadb.org/intal/catalogo/PE/2011/09 063.pdf

Blanco, J. (2010). Notas sobre la relación transporte-territorio: implicancias para la planificación y una propuesta de agenda. Revista Transporte y Territorio, 3. Disponible en: https:// dialnet.unirioja.es/descarga/articulo/3675947.pdf

Bonaudo, M y Godoy, C. (1985). Una corporación y su inserción en el proyecto agroexportador: la Federación Agraria Argentina, 1912-1933. En Anuario, 11 (pp. 151-216). Universidad Nacional de Rosario.

Bonaudo, M. (2006). La historia rural pensada desde una periferia. En J. Gelman (comp.), La historia económica argentina en la encrucijada: balances y perspectivas (pp. 231-246). Buenos Aires: Prometeo Libros.

Bonaudo, M. (2007). Historia o historias rurales: un campo de diálogo entre historiadores. En O. Graciano y S. Lazzaro (comps.), La argentina rural del siglo XX (pp. 15-24). Buenos Aires: La Colmena. 
Campi, M. (2008). Cambios históricos en la frontera agraria pampeana. La tecnología y el uso de la tierra. Tesis de Maestría en Investigación Histórica, Universidad de San Andrés. Disponible en: http://hdl.handle.net/10908/94

Dirección Provincial de Vialidad - DPV (2014). Regionales de conservación caminos de tierra. Departamento planificación, evaluación y control. Cartografía de marzo de 2014. Disponible en: http://www.accpc.com.ar/descargas/Vialidad-Regionales2014.pdf.

Djenderedjian, J. (2008). La colonización agrícola en Argentina, 1850-1900: problemas y desafíos de un complejo proceso de cambio productivo en Santa Fe y Entre Ríos. Revista América Latina en la historia económica, 30, 127-157.

Flichman, G. (1977). La renta del suelo y el desarrollo agrario argentino. Buenos Aires: Siglo XXI Editores.

Fouracre, P. y Dyson, M. (2006). Caminos rurales. Ponencia presentada en el Foro Internacional para el Transporte Rural y el Desarrollo (IFRTD).

Gallo, E. (1983). La pampa gringa. La colonización en Santa Fe, 1870-1895. Buenos Aires: Editorial Sudamericana.

García Voglino (1958). De la delegación de Córdoba. Revista Carreteras, 15, julio - septiembre.

Gelman, J. (2007). Perspectivas de investigación y metodología en la historia agraria, hoy. Algunas reflexiones desde el ayer. En O. Graciano y S. Lazzaro (comps.), La Argentina rural del siglo XX: fuentes, problemas y métodos (pp. 25-32). Buenos Aires: La Colmena

Girbal-Blacha, N. (2001). La historiografía agraria argentina: enfoques microhistóricos regionales para la macrohistoria rural del siglo XX, 1980-1999. Revista Estudios Interdisciplinarios de América Latina y el Caribe, 12(2), 5-34.

Girbal-Blacha, N. (2007). Vigencia de las tramas regionales en la historia de la Argentina rural. En O. Graciano y S. Lazzaro (comps.), La Argentina rural del siglo xx: Fuentes, problemas y métodos. (pp. 41-54). Buenos Aires: La Colmena.

Girbal-Blacha, N. (2013). Historia y memoria rural. Tramas regionales para la construcción de la historia rural argentina. Breves Contribuciones del I.E.G., 24, 118-131.

Graciano, O. y Lazzaro, S. (2007). La Argentina rural del siglo xx: Fuentes, problemas y métodos. Buenos Aires: La Colmena.

Halperin, T. (2005). Vida y muerte de la República verdadera (1910-1930). Buenos Aires: Ariel.

Hora, R. (2015). Los terratenientes de la pampa argentina: una historia social y política 1860-1945. Buenos Aires: Siglo XXI editores.

Hummet, E. (1956). Los caminos vecinales. Raíces del aparato vial. Revista Carreteras, 6, abril-junio.

Lattuada, M. (2006). Acción colectiva y corporaciones agrarias en la Argentina. Transformaciones institucionales a fines del siglo XX. Buenos Aires: Universidad Nacional de Quilmes.

López, M. (2007). Antecedentes ideológicos de la Ley Mitre. En J. Schvarzer, A. Regalsky y T. Gómez (eds.), Estudios sobre la historia de ferrocarriles argentinos 1857-1940 (1 ${ }^{\text {a }}$ ed., pp. 91-120). Buenos Aires: Universidad de Buenos Aires. 
Lordi, H. y Regazzoli, G. (2011). Estudio y análisis de los beneficios económicos provenientes de un plan de rehabilitación y conservación de caminos rurales. Revista Carreteras, 202, junio.

Maldonado, O. (1989). Red de caminos rurales y vecinales en la República Argentina. Conservación por intermedio de Consorcios Camineros. Revista Carreteras, 130, enero-marzo.

Molina, H. (1958): La vialidad en Córdoba. En Revista Carreteras, número 15, Julio - septiembre de 1958.

North, D. (1993). Instituciones, cambio institucional y desempeño económico. México DF: Fondo de Cultura Económica.

Neffa, J. (1998). Modos de regulación, regímenes de acumulación y sus crisis en Argentina, 18801996. Una contribución a su estudio desde la teoría de la regulación. Buenos Aires: Eudeba.

Olivera, G. (2008). Cooperativismo agrario: una nueva área temática en la renovación de la historia rural. En J. Balsa, G. Mateo y M. Ospital (comps.), Pasado y presente en el agro argentino ( $1^{\text {a }}$ ed., pp. 219-235). Buenos Aires: Lumiere.

Ostrom, E. y Ahn, T. (2003). Una perspectiva del capital social desde las ciencias sociales: capital social y acción colectiva. Revista mexicana de sociología, 65(1), 155-233.

Picca, A. (2016). Experiencia de los Consorcios Camineros en la provincia de Córdoba. Ponencia presentada en el Congreso argentino de Caminos Rurales organizado por la Asociación Argentina de Carreteras. Olavarría, provincia de Buenos Aires, 29 y 30 de junio.

Regalsky. A. (2007). Los orígenes de la Ley Mitre. Algunos apuntes críticos. En J. Schvarzer, A. Regalsky y T. Gómez (eds.), Estudios sobre la historia de ferrocarriles argentinos 1857-1940 ( $1^{\text {a }}$ ed., pp. 123-152). Buenos Aires: Universidad de Buenos Aires

Rofman, A. y Villar, A. (2007). Módulo de Formación General: Actores del Desarrollo Local. Proyecto PNUD ARG/04/005 Apoyo a la gestión de la Secretaría de Políticas Sociales y Desarrollo Humano y del Ministerio de Desarrollo Social. Argentina.

Ruffini, M. (2003). Estado nacional y territorios. Notas sobre la economía rionegrina en la etapa de los gobiernos militares, 1884-1898. Revista Mundo Agrario, 3(6). FaHCE, Universidad Nacional de la Plata.

Scalabrini, R. (1974). Historia de los ferrocarriles argentinos, $6^{\mathrm{a}}$ ed. Buenos Aires: Editorial Plus Ultra.

Taylor, C. (1948). Rural Life in Argentina. Baton Rouge: Lousiana State University Press.

Valdemarca, L. (2006). Acción Colectiva y capital social. Institucionalizando la cooperación. Los Cóndores, 1950-1955. En G. Olivera (comp.) Cooperativismo agrario: instituciones, políticas públicas y procesos históricos. (pp 153-181). Córdoba: Ferreyra Editor.

Woolcock, M. (2000). Social capital in theory and practice: reducing poverty in building partnerships between states, markets and civil society. Ponencia presentada en el International Symposium "Social Capital Formation in Poverty Reduction: Which Role for Civil Society and the State" (pp. 20-44). UNESCO. Geneva. June-july. 